\title{
Expression of melanocortin 1 receptor before and after narrowband UVB phototherapy treatment in patients with stable vitiligo: A prospective study
}

\author{
JORGE OCAMPO-GARZA ${ }^{1}$, MAURICIO SALINAS-SANTANDER ${ }^{2}$, OLIVERIO WELSH ${ }^{1}$, \\ MAIRA HERZ-RUELAS ${ }^{1}$ and JORGE OCAMPO-CANDIANI ${ }^{1}$
}

${ }^{1}$ Department of Dermatology, 'Dr. Jose Eleuterio Gonzalez' University Hospital of The School of Medicine,
Universidad Autónoma de Nuevo León, Monterrey, Nuevo León $64460 ;{ }^{2}$ Department of Research,
Universidad Autónoma de Coahuila, Faculty of Medicine, Saltillo, Coahuila 25000, Mexico

Received May 31, 2019; Accepted August 16, 2019

DOI: $10.3892 /$ etm.2020.8435

\begin{abstract}
Vitiligo is a disease characterized by skin depigmentation caused by the selective destruction of melanocytes. The melanocortin system participates as a regulator of melanogenesis and skin pigmentation. Narrowband UVB phototherapy (nb-UVB) is currently considered to be the gold standard and first choice treatment method for vitiligo vulgaris. The aim of the present study was to analyze the clinical and biochemical parameters of vitiligo, as well as to determine the expression of proopiomelanocortin (POMC), melanocortin 1 receptor (MC1R) and melanocortin 4 receptor (MC4R) genes in the skin of patients with stable vitiligo receiving nb-UVB phototherapy. Patient clinical and biochemical parameters, and the skin biopsies of 22 patients with stable vitiligo were analyzed. These biopsies were obtained before and after nb-UVB phototherapy. The genetic expression analysis of POMC, MC1R and MC4R genes was performed via RNA-Sequence analysis. A statistical evaluation of the clinical and biochemical parameters, the degree of response to treatment and the expression profiles of the melanocortin system genes were performed to identify their association with treatment response. A two-sided $\mathrm{P} \leq 0.05$ value was considered to indicate a statistically significant difference. Alterations were observed in the expression profiles of MC1R following nb-UVB phototherapy $(\mathrm{P} \leq 0.05)$. In addition, elevated levels of triiodothyronine were associated with a poor response to nb-UVB phototherapy. In conclusion
\end{abstract}

Correspondence to: Dr Jorge Ocampo-Candiani, Department of Dermatology, 'Dr. Jose Eleuterio Gonzalez' University Hospital of The School of Medicine, Universidad Autónoma de Nuevo León, 2 Consulta Externa, Francisco I Madero poniente y Av. Gonzalitos s/n, Monterrey, Nuevo León 64460, México

E-mail: jocampo2000@yahoo.com.mx

Key words: stable vitiligo, narrowband UVB phototherapy, expression, melanocortin 1 receptor gene, triiodothyronine the current study revealed that nb-UVB phototherapy altered the expression profile of the MC1R gene.

\section{Introduction}

Vitiligo is a depigmentation disease of the skin and mucous membranes caused by the selective destruction or malfunction of melanocytes (1). Genetic and environmental factors have been implicated in vitiligo onset (2). The pathogenesis of vitiligo is controversial; however, the main hypotheses indicate that depigmentation occurs due to destruction of the melanocytes mediated by autoimmunity (3), as well as intrinsic anomalies of melanocytes (4). Currently, evidence suggests that both hypotheses are linked through mechanisms of innate immunity (5).

The melanocortin system is a regulatory module that acts by coordinating and executing the local response to stress. It consists of three melanocortin peptides: Alpha, beta and gamma melanocyte stimulating hormone (alpha-MSH, beta-MSH and gamma-MSH), and adrenocorticotropic hormone (ACTH), which are post-translational products of proopiomelanocortin (POMC) (6). POMC is a protein synthesized in the pituitary gland, and in other parts of the body, such as the skin, where its products play important roles as regulators of melanogenesis and melanocyte survival (7). Skin levels of the POMC and POMC/corticotropin releasing hormone peptides are determined by physiological changes associated with hair cycle (highest in anagen phase), ultraviolet radiation exposure, immune cytokine release, or the presence of cutaneous pathology (8).

The MSH and ACTH proteins bind to melanocortin receptors of extracellular G proteins (MCR), of which five subtypes have been described: Melanocortin receptor 1 (MCR1) to melanocortin receptor 5 (MCR5) (9). The MC1R receptor has shown high cutaneous expression, influencing both the pigmentation and the immune system (10). It participates in the regulation of skin color, in the protective effect of melanin and it has been considered as a susceptibility gene for the development of melanoma $(11,12)$. MCIR receptor has become a new therapeutic target of diseases that involve its participation. 
Afamelanotide is a long-lasting synthetic analog of $\alpha-\mathrm{MSH}$, which binds to the melanocortin-1 receptor and stimulates melanocyte proliferation and melanogenesis $(13,14)$.

In the pigmentation process, MC1R is a key signaling molecule on melanocytes that responds to $\alpha$-MSH by inducing expression of enzymes responsible for eumelanin synthesis, through a complex series of events, mainly mediated by the cAMP signaling cascade, which leads to an increase in tyrosinase activity and activation of biosynthesis of pigments (15). Kingo et al observed that the expression levels of POMC is low and found that expression of MC1R and MC4R was decreased in vitiligo-affected skin compared with non-lesional skin from these patients (6). Additionally, the expression of these receptors was elevated in the non-lesional skin of vitiligo patients compared with the skin of healthy control subjects (6). From these findings, they concluded that the melanocortin system could be implicated in the pathogenesis of vitiligo.

Currently, narrow-band ultraviolet type B light (nb-UVB) is considered the gold standard for treating generalized vitiligo $(16,17)$. Its mechanism of action is through the induction of local immunosuppression, stimulation of the proliferation of melanocytes, induction of melanogenesis in the skin (18), and the production of the melanocyte stimulating hormone (MSH) (17).

Because the effect of nb-UVB phototherapy on gene expression of the melanocortin system has not been studied to date, the aim of this study is to analyse the expression profile of the genes involved in the melanocortin system (POMC, MC1R and MC4R) in the affected and non-affected skin of stable vitiligo patients before and after nb-UVB phototherapy treatment, as well as to analyse the clinical and biochemical variables that may be involved in the treatment response.

\section{Patients and methods}

Subjects. The present study included 22 patients over 18 years of age due to regulation of the Institutional Review Board, with skin photo types II-V of the Fitzpatrick classification (19), with stable vitiligo (in which there is no growth or appearance of new lesions within a period of 6 months), which affects an area greater than $10 \%$ and less than $80 \%$ of the body surface, treated in the Dermatology Department of the University Hospital 'Dr. José E. González', in Monterrey, Mexico, between September 2013 and February 2016. The protocol and informed consent form were approved by the Ethics and Research Committee of the 'Dr. Jose Eleuterio Gonzalez' University Hospital of the School of Medicine and registered the protocol and forms of informed consent under the code DE12-006. Written informed consent was obtained from all the participants. They underwent an interview and clinical evaluation by dermatologists to confirm the diagnosis. The patients didn't received any type of vitiligo treatment during the previous 6 months. Patients with another vitiligo type, with severe/poorly controlled systemic disease, for example type 2 Diabetes Mellitus (T2DM), or with skin cancer or a personal history of skin cancer, and patients who were photosensitive, or pregnant/lactating were excluded.

Demographic information, personal data, history of the disease, age of onset, number and distribution of lesions, height, weight and body mass index (BMI), family history of the disease and association with other autoimmune diseases were recorded.
Phototherapy treatment. Patients were treated with nb-UVB phototherapy Spectra camera Model 311/350 nm (DAAVLIN) at 240 volts, $30 \mathrm{amps}$, and $60 \mathrm{~Hz}$ in 2 sessions per week for a period of 6 months (completing a total of 48 sessions), starting with a dose of $150 \mathrm{~mJ} / \mathrm{cm}^{2}$ with incremental increases of $50 \mathrm{~mJ} / \mathrm{cm}^{2}$ every third session (depending on the skin photo type) until achieving the minimum dose of asymptomatic erythema. The dose was maintained once the clinical response was observed. In patients who presented irritation or intolerance, the dose was reduced to the previously tolerated dose.

The follow-up of the treatment was documented through baseline iconography and at 1,3 and 6 months after the initiation of treatment. Two independent investigators evaluated clinical outcomes (repigmentation changes). The iconographies were taken with a Sony Exmor DSC-HX1 9.1-megapixel camera (Shinagawa-ku).

At the end of $48 \mathrm{nb}-\mathrm{UVB}$ phototherapy sessions, the response to treatment observed in patients was classified into three groups: Low (those subjects whose response to treatment was less than 10\%), medium (those subjects whose response to treatment was between 10 and 29.9\%), and high (those subjects whose response to treatment was greater than $30 \%$ ).

Biological samples. Venous blood samples were collected from each subject to perform laboratory studies: Complete Blood Count (CBC), Comprehensive Metabolic Panel (CMP), Antinuclear Antibodies (ANAs) and Complete Thyroid Profile including levels of Triiodothyronine (T3), L-thyroxine (T4) and thyroid stimulating hormone (TSH).

Four punch biopsies $(4 \mathrm{~mm})$ were performed in each patient: 2 initial biopsies (one of a depigmented skin lesion and one of non-affected-non-perilesional skin, to rule out degenerative changes in melanocytes), and 2 biopsies at the end of nb-UVB phototherapy treatment (one of the treated lesional skin that did not repigment and one of the repigmented skin). The biopsies were stored at $-80^{\circ} \mathrm{C}$ immersed in RNA later (Ambion, Thermo Fisher Scientific, Inc.) for preservation, stabilization and protection of RNA, until they were processed for expression analysis. RNA extraction was performed using RN easy Fibrous Tissue Mini kit (Qiagen, Inc.) following the protocol established by the manufacturer.

The total RNA amount was determined by spectrophotometric quantification in microvolume with Nanodrop equipment (Thermo Fisher Scientific, Inc.) and by quantification by a fluorometric method with Qubit-RNA-BR Assay Kit for Qubit 2.0 (Thermo Fisher Scientific, Inc.). RNA quality was determined by capillary electrophoresis using Experion Automated Electrophoresis System (Bio-Rad Laboratories, Inc.), accessed by the determination of $18 \mathrm{~S} / 28 \mathrm{~S}$ ribosomal RNA ratio.

Expression analysis using TruSeq Targeted RNA. Total RNA $(100 \mu \mathrm{g})$ was extracted from the biopsies of the patients and treated with DNase, and a reverse transcription reaction was performed using the ProtoScript ${ }^{\circledR}$ reverse transcriptase (New England Biolabs), following the indications established for TruSeq Targeted RNA analysis (Illumina, Inc.).

The RNA expression analysis was performed by next-generation sequencing (RNA-Seq) in an Illumina MiSeq sequencer using MiSeq ${ }^{\circledR}$ Reagent kit v3 (150 cycles) and a 
TruSeq ${ }^{\circledR}$ Targeted RNA Custom panel designed to detect the expression profile of 3 genes involved in pigmentation: POMC, MC1R and MC4R.

The data obtained by RNA-Seq were analysed within Base Space (https://basespace.illumina.com) using the TruSeq Targeted RNA tool. The count data for each sample were exported and normalized by equalizing the total number of counts.

Statistical analysis. The sample size was calculated for comparing means, considering the study of melanocortin expression previously conducted by Kingo et al (6) (alpha $=0.05$, standard deviation $=0.002$, mean $=0.008$ and delta $=0.10)$, and it was estimated that a minimum sample size of 19 subjects was sufficient to carry out this study.

The statistical analysis was performed using the statistical package IBM SPSS Statistics version 17.0 (IBM Corp.). Descriptive statistics were used for the demographic data, as well as for all defining variables. To contrast the normality, the Kolmogorov-Smirnov and Shapiro-Wilk tests were carried out. According to the type of distribution presented by the groups (normal or non-normal) of continuous variable, comparisons were made of 2 groups using Student's T-test and Mann-Whitney U-test, respectively. The comparisons of more than two group means were made using one-way analysis of variance (ANOVA) for the variables with normal distribution, and the H-Kruskal-Wallis test for the variables with non-normal distribution. Additionally, post hoc tests were performed for comparison between groups (Tukey and Bonferroni for equal variances, Tamhane and Dunnett T3 for different variances). The association between variables was determined using the chi-square test $\left(\chi^{2}\right)$ and Fisher's exact test for Antinuclear Antibodies and the degree of response to treatment. To determine existing significant differences between expression profiles, multiple comparison Kruskal-Wallis test was used due to the non-normal distribution of the data, and Mann-Whitney U test was used to confirm differences between two independent groups of gene expression profiles. $\mathrm{P}<0.05$ was considered to indicate a statistically significant difference.

\section{Results}

Patient data. In this study, 22 patients with stable vitiligo were included, 8 women (with an average age of $40 \pm 16.43$, minimum of 19 years and a maximum of 70 years) and 14 men (with an average age of $39.79 \pm 12.18$, minimum 24 years, maximum 69 years) with greater than $10 \%$ of their body surface affected by the disease, and skin photo types III-IV (Fitzpatrick classification (19)).

Response to $n b-U V B$ phototherapy. The clinical evaluation determined that all patients had some degree of repigmentation (from 1 to $65 \%$ ) after nb-UVB phototherapy. In this regard, 8 $(36.36 \%)$ subjects demonstrated a low response to treatment (Fig. 1), 8 (36.36\%) displayed a medium response (Fig. 2) and $6(27.28 \%)$ exhibited a high response (Fig. 3).

Effect of $n b-U V B$ phototherapy on various clinical and biochemical parameters. Regarding the biochemical and anthropometric parameters analysed, it can be observed by
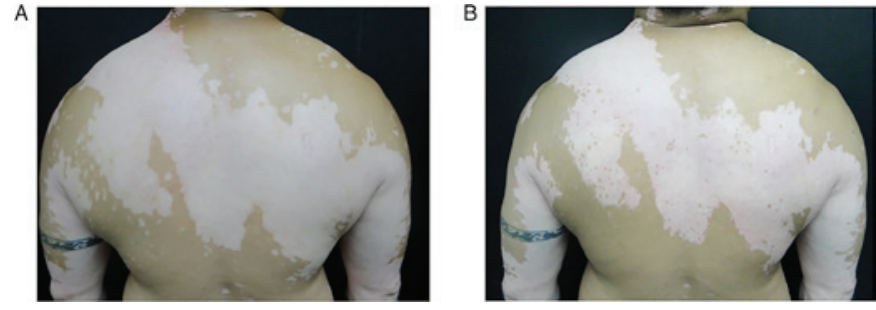

Figure 1. Patients with stable vitiligo that exhibited a treatment response $<10 \%$. (A) Before treatment with nb-UVB phototherapy. (B) After 48 sessions of nb-UVB phototherapy. Nb-UVB, narrowband UVB phototherapy.
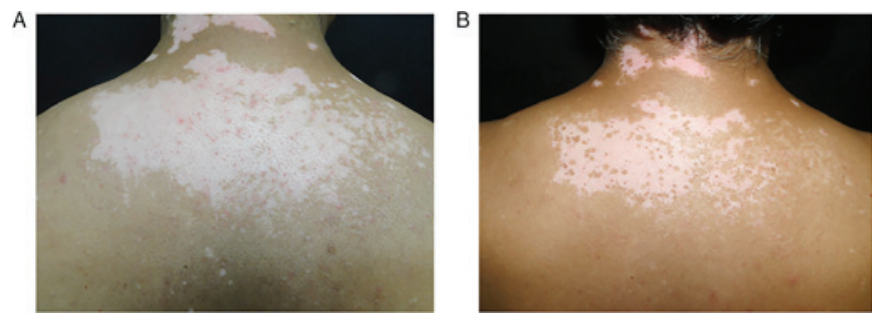

Figure 2. Patients with stable vitiligo that exhibited a treatment response between $10-29.9 \%$. (A) Before treatment with nb-UVB phototherapy. (B) After 48 sessions of nb-UVB phototherapy. Nb-UVB, narrowband UVB phototherapy.
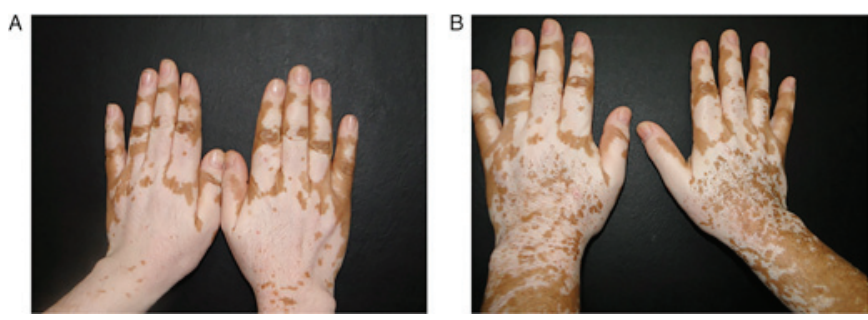

Figure 3. Patients with stable vitiligo that exhibited a treatment response $>30 \%$. (A) Before treatment with nb-UVB phototherapy. (B) After 48 sessions of treatment with nb-UVB phototherapy. Nb-UVB, narrowband UVB phototherapy.

ANOVA analysis that the subjects who displayed a greater response to treatment have, on average, higher levels of T4, and lower levels of T3, TSH, weight and BMI (Table I). Concerning the levels of triiodothyronine (T3), it could be observed that this value is significantly lower in the subjects who presented a high response to treatment, in comparison with those who presented a medium response to treatment $(\mathrm{P}=0.042)$ (Table I).

On the other hand, the chi square test analysis $\left(\chi^{2}\right.$ between the degree of response to treatment and the presence of ANA's (considering as positive those subjects who presented dilutions equal to or greater than 1:80), allowed to determine that there is no association between the presence of antinuclear antibodies and the degree of response to nb-UVB phototherapy $(\mathrm{P}=0.097)$.

Expression analysis. For the expression profile analysis of the POMC, MC1R and MC4R genes, the 4 skin biopsies obtained from the 22 patients included in this study were used. The results by Kruskal-Wallis and Mann-Whitney U test analysis showed: POMC (vitiligo skin biopsy expression $0.79 \pm 0.17$, normal skin biopsy expression $0.71 \pm 0.16$, repigmented skin biopsy expression $0.49 \pm 0.13$, and expression of skin biopsy that did 
Table I. Biochemical and anthropometric parameters according to the degree of response to nb-UVB phototherapy.

\begin{tabular}{lccc}
\hline & \multicolumn{3}{c}{ Degree of response to nb-UVB phototherapy } \\
\cline { 2 - 4 } & & $\begin{array}{c}\text { Medium } \\
(10-29.9 \%)\end{array}$ & High $(>30 \%)$ \\
Parameters & Low $(<10 \%)$ & & \\
\hline T3 (ng/dl) & $147.94 \pm 51.60$ & $129.11 \pm 18.24$ & $104.37 \pm 14.27^{\text {a }}$ \\
T4 $(\mathrm{mcg} / \mathrm{dl})$ & $7.53 \pm 2.52$ & $7.51 \pm 1.00$ & $7.90 \pm 1.10$ \\
TSH (mlU/l) & $2.37 \pm 0.78$ & $2.07 \pm 0.70$ & $1.76 \pm 0.68$ \\
Weight $(\mathrm{kg})$ & $98.69 \pm 14.53$ & $79.72 \pm 25.22$ & $74.17 \pm 13.57$ \\
BMI & $32.50 \pm 4.83$ & $28.79 \pm 8.77$ & $27.55 \pm 3.24$ \\
\hline
\end{tabular}

${ }^{\mathrm{a}} \mathrm{P}=0.042$ vs. Medium (10-29.9\%). Data are presented as the mean \pm standard deviation. $\mathrm{Nb}-\mathrm{UVB}$, narrowband UVB phototherapy; $\mathrm{T} 3$, triiodothyronine; T4, thyroxine; TSH, thyroid-stimulating hormone; BMI, body mass index.

not repigment following treatment $0.48 \pm 0.15$ ); MC1R (vitiligo skin biopsy expression $0.78 \pm 0.16$, normal skin biopsy expression $1.59 \pm 0.16$, repigmented skin biopsy expression $1.29 \pm 0.15$, and expression of skin biopsy that did not repigment following treatment $0.52 \pm 0.12$ ); and MC4R (vitiligo skin biopsy expression no detected, normal skin biopsy expression $0.19 \pm 0.11$, repigmented skin biopsy expression $0.17 \pm 0.09$, and expression of skin biopsy that did not repigment following treatment $0.13 \pm 0.07$ ) (Fig. 4), and a statistically significant difference was obtained in the expression for MC1R gene $(\mathrm{P} \leq 0.05)$. Nevertheless, the expression profiles obtained demonstrated no statistically significant relationship with the degree of response to treatment experienced by the patients $(\mathrm{P}>0.05)$.

\section{Discussion}

Nb-UVB phototherapy has been considered the first choice treatment in vitiligo vulgaris as it is effective and well-tolerated (20). In this study, all patients treated for stable vitiligo displayed some degree of repigmentation, reaffirming the usefulness of nb-UVB phototherapy as a therapeutic alternative in cases of stable vitiligo. The UV radiation can upregulate $\alpha$-MSH receptor (MC1R) expression and activity, POMC expression, and POMC peptide production; include $\alpha$-MSH, $\beta$-endorphin, and ACTH molecules that participate in the regulation of skin pigmentation, to protect skin from UV induced injury, and to modulate skin immune response (21). It's well known that UV light stimulates the production of endothelin-1 (ET-1) and POMC by keratinocytes, factors that can act in a paracrine manner by stimulating the function of melanocytes (22).

$\mathrm{Nb}-\mathrm{UVB}$ phototherapy acts through the induction of local immunosuppression and the stimulation of the proliferation of melanocytes in the skin and in the outer sheath of the hair follicle root; there is also a stimulating effect on melanogenesis and on the production of melanocyte-stimulating hormone (MSH) (17).

Currently, compared with other technologies such as RTq-PCR and microarrays, RNA sequencing (RNA-Seq) using next-generation sequencing (NGS) allows determination of gene expression levels with higher efficiency, lower cost and

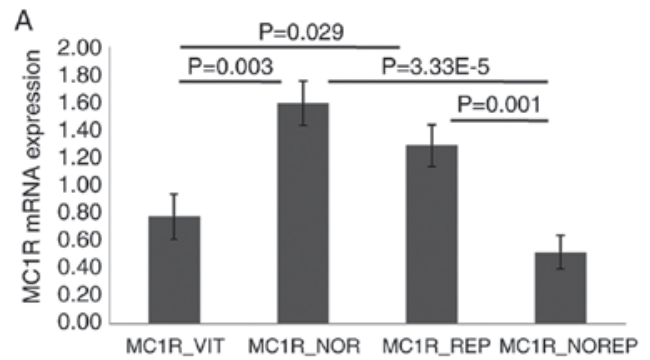

B
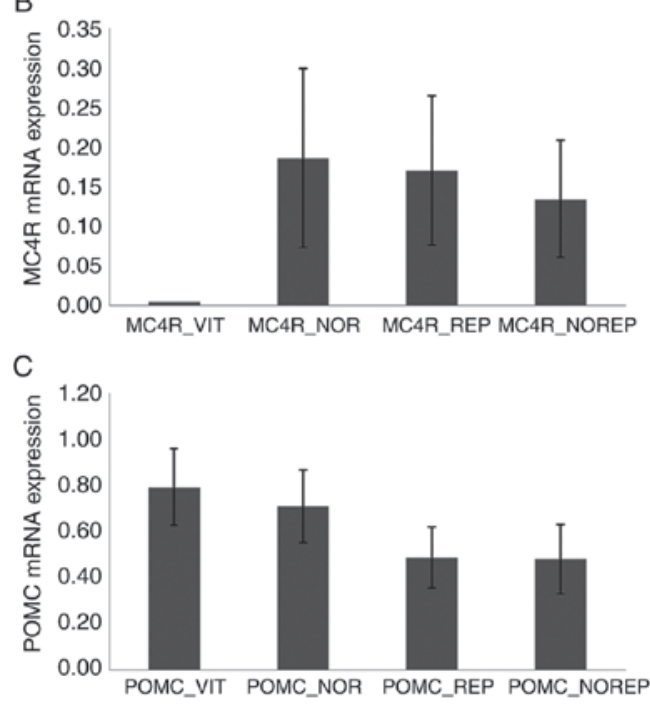

Figure 4. Relative levels of mRNA expression in stable vitiligo skin biopsies (A) Relative levels of MC1R. (B) Relative levels of MC4R. (C) Relative levels of POMC. Data are presented as the mean \pm standard deviation. MC1R, melanocortin 1 receptor; MC4R, melanocortin receptor 4; POMC, proopiomelanocortin; VIT, skin biopsy with vitiligo; NOR, normal skin biopsy; REP, skin biopsy from repigmented skin following treatment; NOREP, skin biopsy that did not repigment following treatment.

shorter times (23). In vitiligo, this technology has already been used to determine the expression profiles in skin biopsies of subjects affected by this disease (24).

Skin pigmentation is due to the pigment produced by melanocytes, eumelanin and pheomelanin, and their accumulation on melanin granules in the keratinocytes. These pigments produced by melanocytes are responsible for skin and hair color. Skin pigmentation is an important protective mechanism against the DNA damaging and mutagenic effects of solar UV radiation (25).

The melanocortin receptor family is a member of the class A rhodopsin-like family of G-protein coupled receptors and consists of five members: MC1R to MC5R; MC1R is found on both melanocytes and leukocytes and its activation promotes UV resistance and anti-inflammatory signaling (26). Melanocortin 1 receptor (MC1R) is a key signaling molecule on melanocytes that responds to $\alpha$-MSH by inducing expression of enzymes responsible for eumelanin synthesis. Moreover $\alpha-\mathrm{MSH} / \mathrm{MC1R}$ signaling is a potent anti-inflammatory pathway and has been shown to promote anti-melanoma immunity (27). Human MC1R genetics variants are associated with sunlight sensitivity (28), and altered expression of MC1R have been associated with red hair and skin cancer risk (29).

The melanocortin system can play an important role in the pathogenesis of vitiligo, and can be affected by this disease, as 
was reported in the studies of Kingo et al (6) and Nagui et al (30). In our study, we analysed the expression profiles of POMC, MC1R and MC4R before and after nb-UVB phototherapy treatment of patients with stable vitiligo. Considering the skin samples obtained from the patients before starting the treatment, we observed that the expression profile for the MC1R and MC4R genes were higher in normal skin biopsies, and these results are consistent with those described by Kingo et al and Nagui et al $(6,30)$. However, for the POMC gene, we found that there is no statistically significant difference between normal skin and skin with vitiligo prior to treatment with nb-UVB phototherapy, unlike what was described by both of these authors. In Fig. 2 we can observe a slight increase in the level of POMC expression in the skin with vitiligo within the analysed subjects. This slight increase could be due to the need of POMC to stimulate pigmentation in the areas affected by vitiligo.

Regarding the expression profiles for the MC1R gene observed after treatment levels of expression in the skin that repigmented after treatment and the levels in the normal skin of the patient before treatment were statistically similar. This allowed us to suppose that this increase in expression accounts for the repigmentation experimented by the skin (Fig. 4). Otherwise, the skin that did not repigment, presented statistically lower levels than normal skin and skin that was repigmented and even lower than the skin with vitiligo onset (Fig. 4).

Concerning the MC4R gene, we observed that the expression levels after treatment were increased in both biopsies. In addition, the expression profiles of skin biopsies that showed repigmentation and normal tissue before treatment were similar. This increase in expression levels of repigmented tissue is related to the response to treatment. In the non-repigmented skin, the low expression profile for this gene would have been expected, as was observed in the case of MC1R. However, the result obtained leads us to consider two possibilities: (a) that this skin will repigment and there was not enough treatment time to see a positive clinical response; or (b) that this gene is not fundamental in pigmentation, and its expression levels are very low. As already described in the study of Millington (2006) the melanocortin 4 receptor (MC4R) is expressed mainly in the brain, spinal cord, muscle and adipose tissue, with its main function being the regulation of energy metabolism $(10,31)$.

Regarding the biochemical parameters and the response to treatment experimented by patients, it was observed that there is a relationship between the levels of T3 and the response to treatment (Table I). In particular, in the subjects who presented a high response to treatment (greater than $30 \%$ repigmentation), the levels of this hormone were found to be decreased $(104.37 \pm 14.27 \mathrm{ng} / \mathrm{dl})$ compared to the groups of patients who displayed a low or medium response to treatment, which exhibited substantially higher levels of this hormone. In a retrospective study conducted by Subba et al, alterations in the levels of T3, T4 and TSH in subjects affected by vitiligo were described (32). In a study conducted in a Turkish population, high levels of T3 and T4 were described in subjects with different types of vitiligo; and progression of this disease was seen in comparison with control subjects (33).

van Beek et al (2008) described that T4 up-regulates the proliferation of hair matrix keratinocytes and prolonged the duration of the hair growth phase (anagen) in vitro with human hair follicles, whereas their apoptosis is down-regulated by T3 and T4; on the other hand, both T3 and T4, significantly stimulated intrafollicular melanin synthesis (34). The association between thyroid problems and vitiligo is known, mainly in people with hypothyroidism (35), supporting the theory of a thyroid dysfunction and the participation of autoimmune processes in the development of this pathology (36).

In conclusion, $\mathrm{Nb}-\mathrm{UVB}$ phototherapy is effective in the treatment of patients with stable vitiligo, favouring repigmentation and producing changes in expression profiles of three genes involved in the melanocortin system, POMC, MC1R and $\mathrm{MC} 4 \mathrm{R}$, related to the repigmentation process in stable vitiligo patients, mainly MC1R $(\mathrm{P}<0.05)$. Although we reaffirm that the melanocortin system intervenes in the pathogenesis of stable vitiligo, additional studies are required to determine the performance of these genes in other variants of this disease. It is also necessary to evaluate the biochemical parameters of vitiligo patients, mainly T3 levels, since elevated levels of this hormone may lead to a poor response to phototherapy.

\section{Acknowledgements}

Not applicable.

\section{Funding}

The present study was funded by the Department of Dermatology, 'Dr. Jose Eleuterio Gonzalez' University Hospital of the School of Medicine, Monterrey, Nuevo Leon, Mexico.

\section{Availability of data and materials}

The datasets used and/or analyzed during the present study are available from the corresponding author on reasonable request.

\section{Authors' contributions}

JOG, JOC and OW conceived the current study. JOG, JOC, OW and MSS designed the present study. JOG and MHR selected the patients. JOG, MHR and MSS analyzed the data. JOG, MSS interpreted the data. JOG, JOC, OW, MHR and MSS drafted and critically revised the manuscript. JOG, JOC, OW and MSS improved the manuscript. All authors approved the final version of the manuscript.

\section{Ethics approval and consent to participate}

The University Hospital-UANL Institutional Review Board approved and registered the study under the trial number, DE12-006.

\section{Patient consent for publication}

All patients consented to the publication of their data and associated images.

\section{Competing interests}

The authors declare that they have no competing interesets. 


\section{References}

1. Picardo M, Dell'Anna ML, Ezzedine K, Hamzavi I, Harris JE, Parsad D and Taieb A: Vitiligo. Nat Rev Dis Primers 1: 15011, 2015.

2. Shen C, Gao J, Sheng Y, Dou J, Zhou F, Zheng X, Ko R, Tang X, Zhu C, Yin X, et al: Genetic susceptibility to vitiligo: GWAS approaches for identifying vitiligo susceptibility genes and loci. Front Genet 7: 3, 2016

3. Sandoval-Cruz M, García-Carrasco M, Sánchez-Porras R, Mendoza-Pinto C, Jiménez-Hernández M, Munguía-Realpozo P and Ruiz-Argüelles A: Immunopathogenesis of vitiligo. Autoimmun Rev 10: 762-765, 2011.

4. Schallreuter KU, Bahadoran P, Picardo M, Slominski A, Elassiuty YE, Kemp EH, Giachino C, Liu JB, Luiten RM, Lambe T, et al: Vitiligo pathogenesis: Autoimmune disease, genetic defect, excessive reactive oxygen species, calcium imbalance, or what else? Exp Dermatol 17: 139-160, 2008.

5. Richmond JM, Frisoli ML and Harris JE: Innate immune mechanisms in vitiligo: Danger from within. Curr Opin Immunol 25: 676-682, 2013

6. Kingo K, Aunin E, Karelson M, Philips MA, Rätsep R, Silm H, Vasar E, Soomets U and Kõks S: Gene expression analysis of melanocortin system in vitiligo. J Dermatol Sci 48: 113-122, 2007.

7. Rousseau K, Kauser S, Pritchard LE, Warhurst A, Oliver RL, Slominski A, Wei ET, Thody AJ, Tobin DJ and White A: Proopiomelanocortin (POMC), the ACTH/melanocortin precursor, is secreted by human epidermal keratinocytes and melanocytes and stimulates melanogenesis. FASEB J 21: 1844-1856, 2007.

8. Slominski A, Wortsman J, Luger T, Paus R and Solomon S: Corticotropin releasing hormone and proopiomelanocortin involvement in the cutaneous response to stress. Physiol Rev 80: 979-1020, 2000

9. Yang Y: Structure, function and regulation of the melanocortin receptors. Eur J Pharmacol 660: 125-130, 2011.

10. Millington GW: Proopiomelanocortin (POMC): The cutaneous roles of its melanocortin products and receptors. Clin Exp Dermatol 31: 407-412, 2006.

11. Yamaguchi Y, Brenner M and Hearing VJ: The regulation of skin pigmentation. J Biol Chem 282: 27557-27561, 2007.

12. Wolf Horrell EM, Boulanger MC and D'Orazio JA: Melanocortin 1 receptor: Structure, function, and regulation. Front Genet 7: 95, 2016.

13. Tafreshi N, Tichacek CJ, Pandya DN, Doligalski ML, Budzevich MM, Kil H, Bhatt NB, Kock ND, Messina JL, Ruiz EE, et al: Melanocortin 1 receptor targeted $\alpha$-particle therapy for metastatic Uveal melanoma. J Nucl Med 60: $1124-1133,2019$

14. Zubair R, Lyons AB, Vellaichamy G, Peacock A and Hamzavi I: What's new in pigmentary disorders. Dermatol Clin 37: 175-181, 2019.

15. García-Borrón J, Abdel-Malek Z and Jiménez-Cervantes C: MC1R, the cAMP pathway, and the response to solar UV: Extending the horizon beyond pigmentation. Pigment Cell Melanoma Res 27: 699-720, 2014.

16. Vázquez-Martínez OT, Velásquez-Arenas L, Méndez-Olvera N and Ocampo-Candiani J: Vitiligo. Overview and current therapeutics. DCMQ 3, 2006.

17. Majid I: Vitiligo management: An update. BJMP 3: 6, 2010.

18. Carsberg CJ, Warenius HM and Friedmann PS: Ultraviolet radiation-induced melanogenesis in human melanocytes. Effects of modulating protein kinase C. J Cell Sci 107: 2591-2597, 1994

19. Fitzpatrick TB: The validity and practicality of sun-reactive skin types I through VI. Arch Dermatol 124: 869-871, 1988.
20. Stinco G, Trevisan G, Buligan C, Gregoraci G, De Marchi S, di Meo N and Patrone P: Narrow band-ultraviolet B versus clobetasol propionate foam in the treatment of vitiligo: A retrospective study. Dermatol Ther (Heidelb) 3: 95-105, 2013.

21. Slominski AT, Zmijewski MA, Plonka PM, Szaflarski JP and Paus R: How UV light touches the brain and endocrine system through skin, and why. Endocrinology 159: 1992-2007, 2018.

22. Costin GE and Hearing VJ: Human skin pigmentation: Melanocytes modulate skin color in response to stress. FASEB J 21: 976-994, 2007.

23. Wang Z, Gerstein M and Snyder M: RNA-Seq: A revolutionary tool for transcriptomics. Nat Rev Genet 10: 57-63, 2009.

24. Salinas-Santander M, Trevino V, De la Rosa-Moreno E, Verduzco-Garza B, Sanchez-Dominguez CN, Cantu-Salinas C, Ocampo-Garza J, Lagos-Rodríguez A, Ocampo-Candiani J and Ortiz-López R: CAPN3, DCT, MLANA and TYRP1 are overexpressed in skin of vitiligo vulgaris Mexican patients. Exp Ther Med 15: 2804-2811, 2018.

25. Swope VB and Abdel-Malek ZA: MC1R: Front and center in the bright side of dark eumelanin and DNA repair. Int J Mol Sci 19: pii: E2667, 2018.

26. Wolf Horrell EM, Boulanger MC and D'Orazio JA: Melanocortin 1 receptor: Structure, function and regulation. Front Genet 7: 95, 2016.

27. Nasti $\mathrm{T}$ and Timares L: MC1R, eumelanin and pheomelanin: Their role in determining the susceptibility to skin cancer. Photochem Photobiol 91: 188-200, 2015.

28. Hernando B, Sanz-Page E, Pitarch G, Mahiques L, Valcuende-Cavero F and Martinez-Cadenas C: Genetic variants associated with skin photosensitivity in a southern European population from Spain. Photodermatol Photoimmunol Photomed 34: 415-422, 2018.

29. Beaumont KA, Newton RA, Smit DJ, Leonard JH, Stow JL and Sturm RA: Altered cell surface expression of human MC1R variant receptor alleles associated with red hair and skin cancer risk. Hum Mol Genet 14: 2145-2154, 2005.

30. Nagui NA, Mahmoud SB, Abdel Hay RM, Hassieb MM and Rashed LA: Assessment of gene expression levels of proopiomelanocortin (POMC) and melanocortin-1 receptor (MC1R) in vitiligo. Australas J Dermatol 58: e36-e39, 2017.

31. You P, Hu H, Chen Y, Zhao Y, Yang Y, Wang T, Xing R, Shao Y, Zhang W, Li D, et al: Effects of melanocortin 3 and 4 receptor deficiency on energy homeostasis in rats. Sci Rep 6: 34938, 2016

32. Subba K, Karn D and Khatri R: Triiodothyronin, thyroxine and thyrotropin in vitiligo. Kathmandu Univ Med J (KUMJ) 9: 7-10, 2011.

33. Arıcan Ö, Şaşmaz S and Çetinkaya A: Role of thyroid hormones in vitiligo type and progression. Turkderm 37: 269-273, 2003.

34. van Beek N, Bodó E, Kromminga A, Gáspár E, Meyer K, Zmijewski MA, Slominski A, Wenzel BE and Paus R: Thyroid hormones directly alter human hair follicle functions: Anagen prolongation and stimulation of both hair matrix keratinocyte proliferation and hair pigmentation. J Clin Endocrinol Metab 93: 4381-4388, 2008.

35. Gupta Y and Ammini AC: Vitiligo, hypothyroidism and cardiomyopathy. Indian J Endocrinol Metab 16: 463-465, 2012.

36. Kasumagic-Halilovic E, Prohic A, Begovic B and Ovcina-Kurtovic N: Association between vitiligo and thyroid autoimmunity. J Thyroid Res 2011: 938257, 2011.

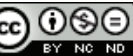

This work is licensed under a Creative Commons Attribution-NonCommercial-NoDerivatives 4.0 International (CC BY-NC-ND 4.0) License. 